\title{
Passive Infrared Sensing Using Plasmonic Resonant Dust Particles
}

\author{
Mark Mirotznik, ${ }^{1}$ William Beck, ${ }^{2}$ Kimberly Olver, ${ }^{2}$ John Little, ${ }^{2}$ and Peter Pa ${ }^{1}$ \\ ${ }^{1}$ Department of Electrical and Computer Engineering, University of Delaware, 106 Evans Hall, Newark, DE 19716, USA \\ ${ }^{2}$ Sensors and Electron Devices Directorate, U.S. Army Research Laboratory, Adelphi, MD 20783, USA
}

Correspondence should be addressed to Mark Mirotznik, mirotzni@ece.udel.edu

Received 5 May 2012; Accepted 24 June 2012

Academic Editor: Georgios Veronis

Copyright ( $\odot 2012$ Mark Mirotznik et al. This is an open access article distributed under the Creative Commons Attribution License, which permits unrestricted use, distribution, and reproduction in any medium, provided the original work is properly cited.

\begin{abstract}
We present computational and experimental results of dust particles that can be tuned to preferentially reflect or emit IR radiation within the $8-14 \mu \mathrm{m}$ band. The particles consist of thin metallic subwavelength gratings patterned on the surface of a simple quarter wavelength cavity. This design creates distinct IR absorption resonances by combining the plasmonic resonance of the grating with the natural resonance of the cavity. We show that the resonance peaks are easily tuned by varying either the geometry of the grating or the thickness of the cavity. Here, we present a computational design algorithm along with experimental results that validate the design methodology.
\end{abstract}

\section{Introduction}

Most objects, either manmade or found in nature, reflect and emit infrared (IR) radiation in a relatively smooth spectrum; however, by applying structures with resonant absorption to the surface of those materials, the reflection and emission spectra can be enhanced or reduced at particular wavelengths (as illustrated in Figure 1). Moreover, by mixing small resonant particles $(<100 \mu \mathrm{m})$ designed for several different wavelengths, we can create IR dust that reflects or emits with a characteristic spectral signature. Such material-bydesign particles would be useful for a variety of practical applications. For example, when applied to a base surface, the resonant particles could be used to tune an IR reflectance to mimic other natural or manmade surfaces. This could be useful as a calibration standard for hyperspectral imaging systems. Additionally, if the particles are chemically functionalized, there are a number of remote atmospheric sensing applications that could be explored.

\section{Infrared Absorbers Using Plasmonic Gratings}

It is well known that metallic surfaces patterned on a subwavelength scale exhibit unusual electromagnetic properties at optical wavelengths. In particular, the presence of localized surface plasmon resonances creates well-defined absorption bands. This phenomenon has been studied and exploited by a number of investigators to realize new types of sensors, optical filters, and absorbers [1-5]. The goal of this work was to numerically and experimentally study plasmonic-based resonant absorbers in the long-wavelength IR (LWIR) band $(8-14 \mu \mathrm{m})$ that could be fashioned into small $(\sim 100 \mu \mathrm{m} \times$ $100 \mu \mathrm{m} \times 25 \mu \mathrm{m})$ dust particles. The dust particles could then be used to tailor the reflectivity/emissivity of a surface or dispersed in air and used for atmospheric sensing applications.

There are a number of small resonant absorbing "dust like" structures that could be used to preferentially absorb, and thus thermally emit, IR radiation at specific wavelengths including dielectric ring resonators, resonant patch antennas, and plasmonic-based resonator. These various structures were compared based on (1) their ability to efficiently absorb IR energy at selected wavelengths within the $8-14 \mu \mathrm{m}$ band, (2) the ability to easily tune the resonant absorption, (3) ease of fabrication, and (4) manufacturing cost. Based on these criteria, we chose to investigate, in detail, the relatively simple surface plasmon-based designs shown in Figure 2. The building blocks for this design are two thin resonant 


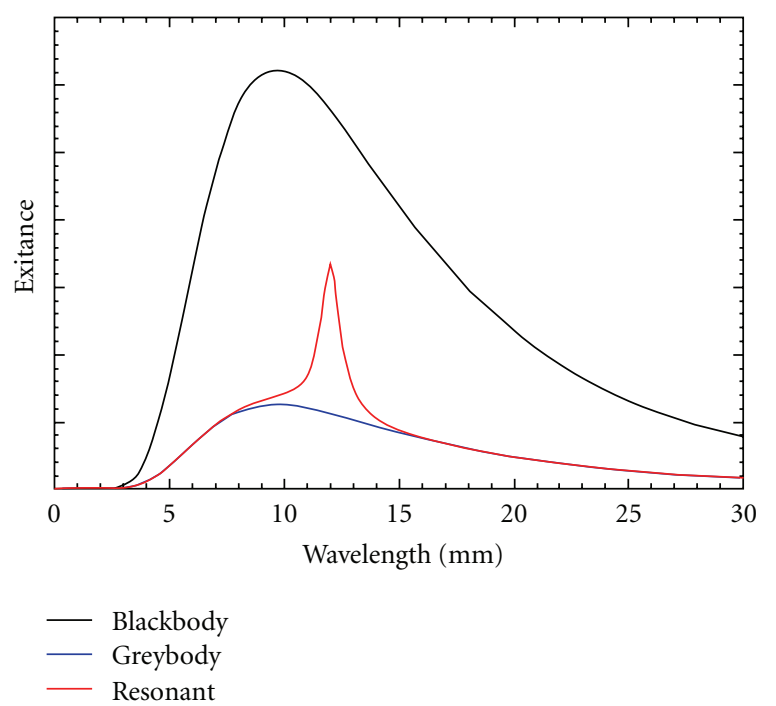

FIgURE 1: Notional diagram that illustrates the normally smooth thermal exitance curves from blackbody and graybody objects compared to the resonant behavior of our "engineered" IR resonant dust.

cavities, one on the top of Figure 2 and the other on the bottom. Each cavity is composed of a thin gold ground plane, a thin dielectric substrate layer (formed from zinc selenide (ZnSe) in our design), and a subwavelength metallic grating made from gold. In the middle of the structure is a relatively thick silicon layer needed for mechanical rigidity. The symmetry of the top and bottom layers was needed since the particles, when dispersed, would orient themselves randomly.

The strong resonant behavior of this design is due to a combination of two different resonant phenomena. The first is a surface plasmon resonance that is excited within the subwavelength gold grating. The second is a cavity resonance excited in the ZnSe substrate region that is between the grating layer and the metallic ground plane layer. By adjusting the thickness of the ZnSe substrate for a given grating period and duty cycle, a strong absorption resonance can be excited at any wavelength within the 8-14 micron band. To create small dust particles, a large sample is diced into small $(\sim 100 \mu \mathrm{m} \times 100 \mu \mathrm{m} \times 25 \mu \mathrm{m})$ particles.

\section{Computational Modeling and Design}

Two different computational models were employed to rigorously design and validate the resonant structure shown in Figure 2. The first method is a fully periodic planar method called the rigorous coupled wave method. The second method, finite element method (FEM), was used to investigate finite-sized particle effects. A brief description of these two methods along with simulation results are presented in the next two sections.

3.1. Modeling of Infinitely Periodic Structures Using Rigorous
Coupled Wave Analysis. Two approaches are used extensively for simulating the electromagnetic properties of infinitely periodic subwavelength gratings. The first uses effective media theory to provide closed-form approximations for the effective dielectric constants as a function of the grating structure [6]. Although attractive from a computational perspective, the approximate expressions are accurate only for gratings whose period is much smaller than the wavelength of illumination. As the grating period approaches the wavelength, which is referred to as the resonance regime, the assumptions on which these closed-form expressions are based are no longer valid. For our designs, we assumed grating periods only slightly smaller than the material wavelength and thus could not accurately utilize effective media theory.

We instead employed a second approach using a rigorous electromagnetic model. Although computationally more difficult, this approach is capable of generating accurate results for gratings of any period size and shape. Several rigorous electromagnetic models can be used for this calculation. We chose the rigorous coupled wave (RCW) algorithm originally presented by Moharam and Gaylord [7]. Our specific implementation is based on the enhanced transmittance matrix approach introduced by Moharam and Gaylord [7] and later refined by Lalanne [8] and Noponen and Turunen [9]. For the sake of brevity, we refer the reader to the references above for details on the RCW method. While being accurate, the RCW method does assume the grating structure, shown in Figure 2, is infinite in the transverse directions. The effect of finite-sized samples is investigated in Section 3.3.

\subsubsection{RCW Simulation Results for Infinitely Periodic Surfaces.} Figure 3 presents typical simulation, results calculated using the RCW method. In the figure, the reflectivity of the sample is calculated as a function of wavelength and polarization for a normally Incident Planewave.

For this simulation the ZnSe substrate thickness was assumed to be $1.8 \mu \mathrm{m}$, the gold grating period was $3.0 \mu \mathrm{m}$ with a $50 \%$ duty cycle. The gold gratings were assumed to be $100 \mathrm{~nm}$ thick, and the gold ground planes were $300 \mathrm{~nm}$ thick. The electromagnetic material properties of the gold were determined using the model given in [10]. For the ZnSe layer, a lossless index of refraction of $n=2.41$ was used in all simulations. The incident field was assumed to be normally incident from the top. For this design, a very strong resonance absorption, near-perfect absorption, is predicted near $9.5 \mu \mathrm{m}$ for the case of parallel polarization (Efield polarized along the axis of the grating) and only weak resonances occur for the case of perpendicular polarization (E-field polarized perpendicular to the axis of the grating).

\subsubsection{Reflectance Sensitivity to Geometrical Parameters.} Given a specific substrate and metallization material, such as ZnSe and gold, the dust particle's reflectance can be tuned by proper selection of the geometrical parameters shown in Figure 2: specifically, (1) thickness of the ZnSe layer, denoted by $\mathrm{h}$ in Figure 2; (2) grating period, denoted by $\Lambda$ in Figure 2; (3) the grating's duty cycle given by $(w / \Lambda$ in 


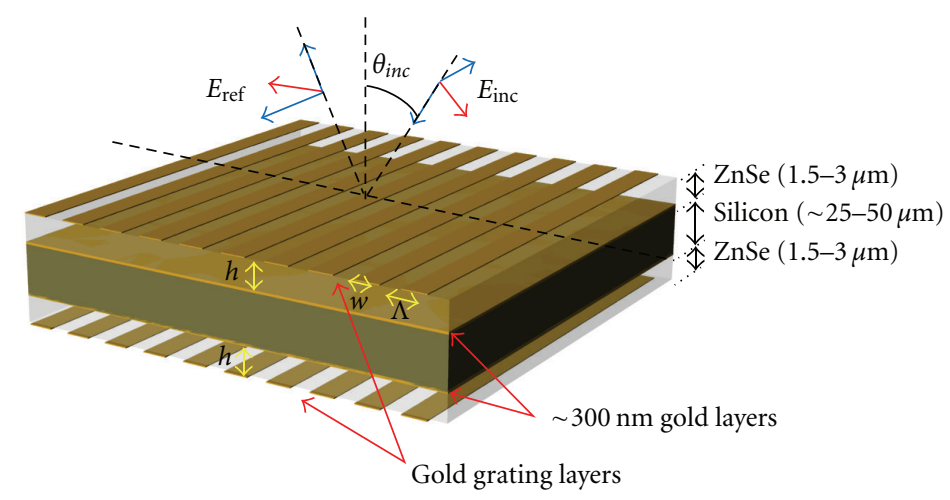

FIGURE 2: Illustration of our surface plasmon-based IR resonant particles. The gold subwavelength gratings along with cavity resonances produce distinct resonant absorption phenomenon within the LWIR band.

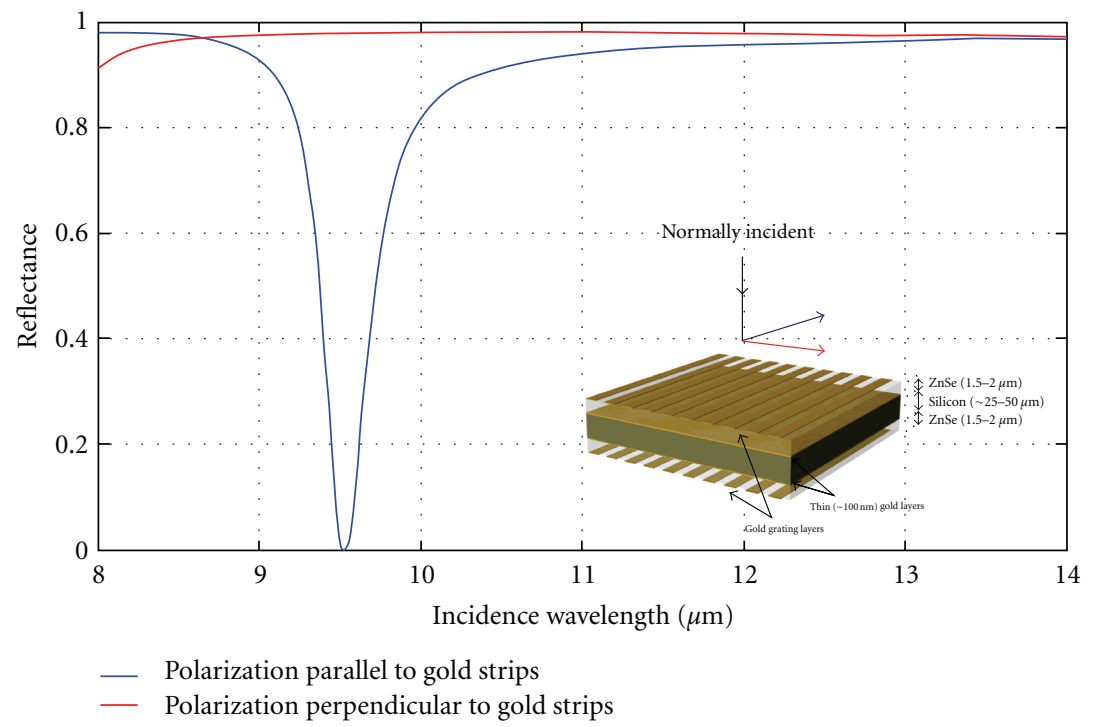

FIGURE 3: Simulation results, using the RCW method, that present the reflectivity at normal incidence within the LWIR band. The reflectivity as expected is polarization dependent due to the anisotropic nature of the gratings.

Figure 2); (4) thickness of the gold grating layer and gold ground plane. Assuming the gold layers are thick enough to prevent transmission (i.e., much thicker than the penetration depth), the variables given by $1-3$ above will have the most effect on the LWIR reflectance.

In Figure 4, we present the effect of the ZnSe substrate thickness on the resonant behavior. As the thickness is increased from 1.5 to $2.5 \mu \mathrm{m}$, the resonant dip shifts from 8.3 to $13.2 \mu \mathrm{m}$, respectively. Thus the resonant behavior can be tuned by simply varying the thickness of the ZnSe substrate.

Alternatively, for a given substrate thickness, the resonant absorption characteristics can be tuned by varying the grating period and duty cycle. Shown in Figure 5 is the simulated reflectance of a sample in which the substrate thickness was fixed at $2.0 \mu \mathrm{m}$ and the grating period was varied from 1.0 to $3.0 \mu \mathrm{m}$. For this simulation, the duty cycle was fixed at $50 \%$. While the resonant wavelength clearly varied with grating period, the change was less sensitive than varying substrate thickness. Moreover, by just changing the grating period, with all other parameters fixed, the amplitude of the resonance would vary considerably. Lastly, we varied the grating's duty cycle while holding the substrate thickness and grating period fixed at 2.0 and $3.0 \mu \mathrm{m}$, respectively. As shown in Figure 6, the grating duty cycle has a large effect on not only the resonant wavelength but also on the amplitude and bandwidth of the resonance.

The sensitivity to incident angle was also evaluated using the RCW code. A typical result for the case of both parallel and perpendicular polarization is shown in Figure 7. Here, the simulation results predict that the resonant frequency for parallel polarization (Figure 7(a)) should slowly increase as the incident angle increases from normal incidence $(0$ degrees in the figure) to near grazing angles (80 degrees). It is interesting to note that for the case of parallel polarization (Figure 7(a)) the variation in resonant wavelength with incident angle is relatively small $(<1 \mu \mathrm{m})$ even with near-grazing incident angles. For the given application of resonant dust particles, this is an attractive feature since the orientation of 


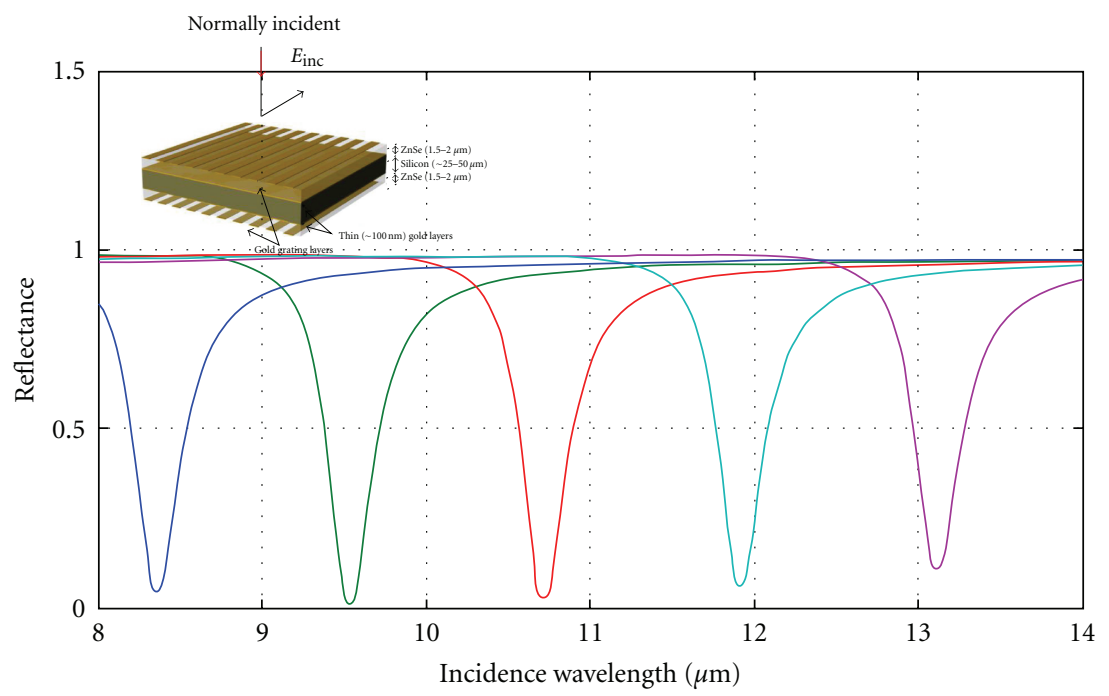

\footnotetext{
ZnSe substrate thickness $=1.5$ microns - ZnSe substrate thickness $=2.25$ microns
- ZnSe substrate thickness $=1.75$ microns - ZnSe substrate thickness $=2.5$ microns

_ ZnSe substrate thickness $=2$ microns
}

FIGURE 4: Simulation results, using the RCW method, that present the reflectance at normal incidence within the LWIR band as the ZnSe substrate thickness is varied from 1.5 to $2.5 \mu \mathrm{m}$. For this simulation, the grating period is fixed at $3.0 \mu \mathrm{m}$ with a $50 \%$ duty cycle. The incident wave was normally incident with parallel polarization. As the substrate thickness is increased, the resonant absorption peak shifts to longer wavelengths but still remains strong. The bandwidth of the resonance also remains relatively fixed as the substrate thicknesses is varied.

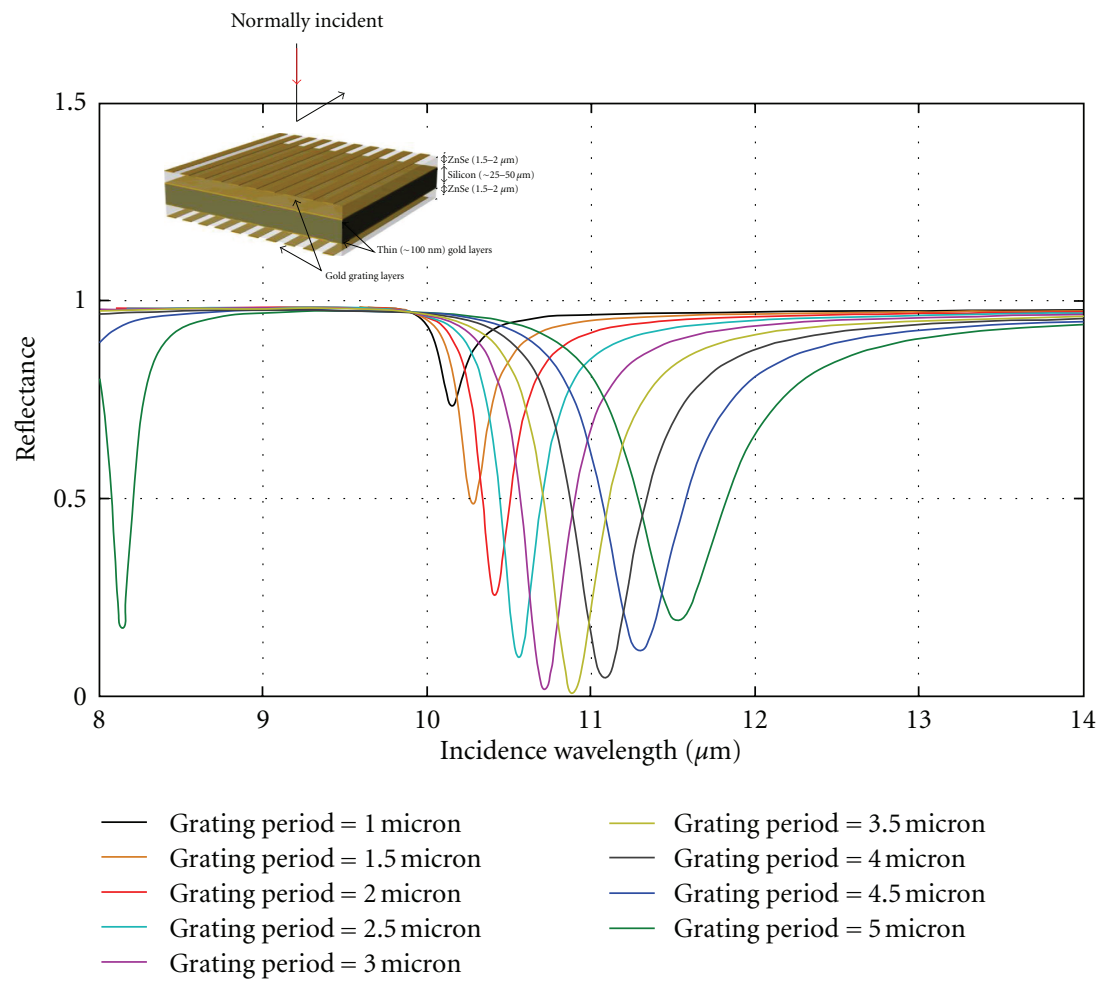

FIGURE 5: Simulation results, using the RCW method, that present the reflectance at normal incidence within the LWIR band as the gold grating period is varied from 1.0 to $5.0 \mu \mathrm{m}$. For this simulation, the substrate thickness is fixed at $2.0 \mu \mathrm{m}$ with a grating duty cycle of $50 \%$. The incident wave was normally incident with parallel polarization. 


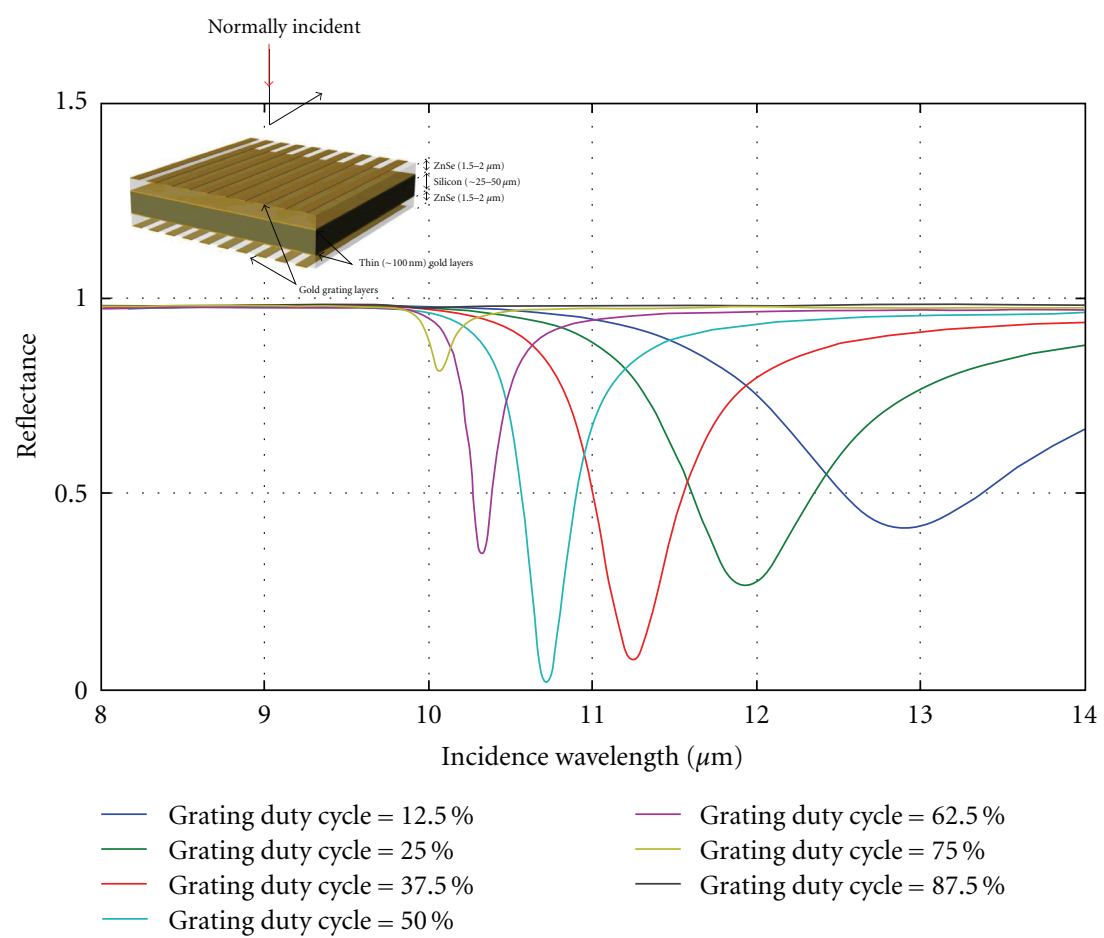

FIGURE 6: Simulation results using the RCW method that present the reflectance at normal incidence within the LWIR band as the duty cycle of the gold grating period is varied from $12.5 \%$ to $87.5 \%$. For this simulation, the substrate thickness is fixed at $2.0 \mu \mathrm{m}$ with a grating period of $3.0 \mu \mathrm{m}$. The incident wave was normally incident with parallel polarization.

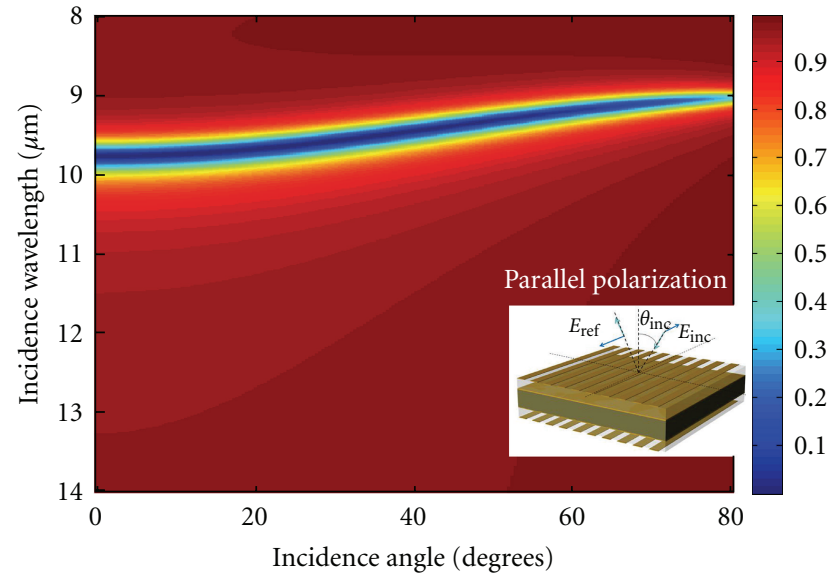

(a)

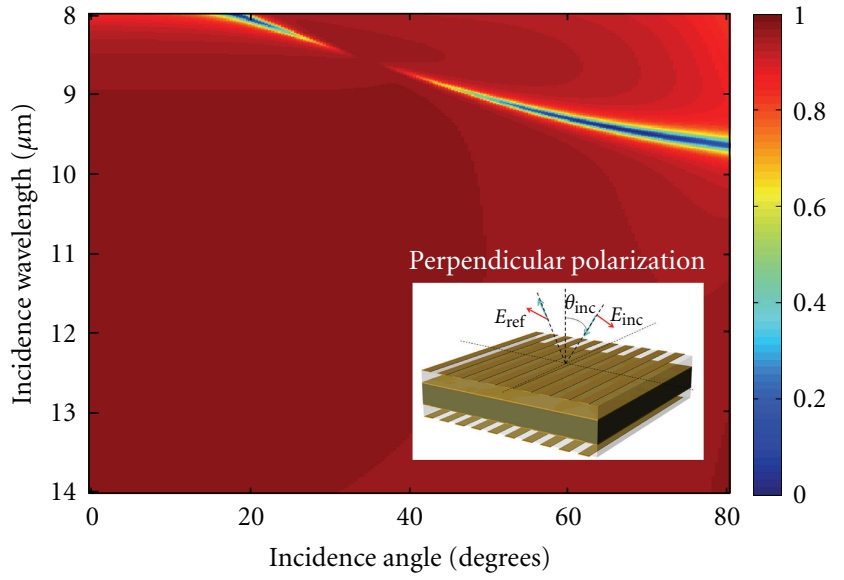

(b)

FIGURE 7: RCW predictions illustrating the sensitivity of our resonant structure with incident angle. The plot on the left (a) is for parallel polarization, while the plot on the right (b) is for perpendicular polarization.

the particles with respect to the incident field cannot be well controlled.

\subsection{Iterative Design. As Figures 4 through 7 demonstrate,} the resonant absorption properties of the structure shown in Figure 2 have a complicated dependence on a number of geometrical parameters. As a result, it is unlikely that any simple analytical design equation could be derived and used to determine an optimal structure for a given desired response.
Consequently, we implemented a numerical iterative design algorithm. Here the RCW method is used to calculate the full wave solution for the reflectance as a function of wavelength, polarization, and angle of incidence for a geometry of a given substrate thickness, grating period, and duty cycle. An optimization algorithm is then used to refine the geometry until an objective function is minimized. The objective function may vary depending on the application, but in most cases we chose to minimize the total reflectance over some desired 
wavelength band. A number of iterative optimization algorithms could be employed including traditional derivativebased algorithms, genetic algorithms, or direct pattern search algorithms. An advantage of both genetic and pattern search algorithms is that they do not require derivatives, and as a consequence work well on nondifferentiable, stochastic, and discontinuous objective functions. Both simple genetic algorithms and direct pattern search algorithms were implemented and tested for the application of interest here. While both methods produced comparable results, the pattern search algorithm was often computationally less expensive.

\subsection{Modeling of Finite Grating Effects Using the Finite Element} Method. The RCW method, while accurate and computationally efficient, assumes the gratings to be infinitely periodic. For our application, the samples are actually diced into small $(\sim 100 \mu \mathrm{m} \times 100 \mu \mathrm{m} \times 25 \mu \mathrm{m})$ particles. Consequently, it is important to understand the effects of relatively small ( $<10$ wavelengths) finite-sized particles on the overall effectiveness of the design. To conduct these simulations we used the commercial EM solver, HFSS from Ansys. Simulations were conducted using HFSS's FEM solver with grating structures that varied from 25 to $100 \mu \mathrm{m}$ on a side.

Figure 8 plots the simulated current density on the surface of a $50 \mu \mathrm{m} \times 50 \mu \mathrm{m} \times 5 \mu \mathrm{m}$ plasmonic particle at a fixed incident wavelength of $10 \mu \mathrm{m}$. The spatial distribution of current is a direct consequence of its finite lateral size and will affect the total absorbed energy. In Figure 9, we plot the average reflectance of the same particle as a function of wavelength. While the total absorption is slightly less and the resonance wavelength is slightly shifted towards longer wavelength, the finite-sized particles still behave with the same general absorption characteristics as the infinitely periodic predictions described previously.

\section{Experimental Fabrication}

To fabricate the samples, a thin (80 microns) 2-inch silicon wafer was first mounted onto a 3-inch (350-500 micron) silicon carrier wafer using Aquabond 55 Adhesive Products wax. The carrier wafer was placed on a hot plate at a temperature of $80^{\circ} \mathrm{C}$. A small amount of wax was smeared on the surface starting at the center and working outward. The thin silicon wafer was carefully placed on top of the wax. A flat glass plate was placed on top of the thin wafer, followed by a brass weight. This was to keep the silicon wafer as flat as possible during the mounting procedure. The hot plate was turned off, and the wax was allowed to cool to room temperature. Excess wax on and around the mounted silicon wafer was removed by gently swabbing it away with a $1 \%$ solution of Aqua Clean. The wafer assembly was placed in a vacuum electron beam evaporator. A blanket metallization of $300 \AA$ of chromium followed by $2000 \AA$ of gold was evaporated on to the wafer. The assembled structure was then moved to another vacuum e-beam evaporator, and a 1.8-micron-thick layer of $\mathrm{ZnSe}$ was evaporated onto the surface. Depositions were performed at $145^{\circ} \mathrm{C}$, with a

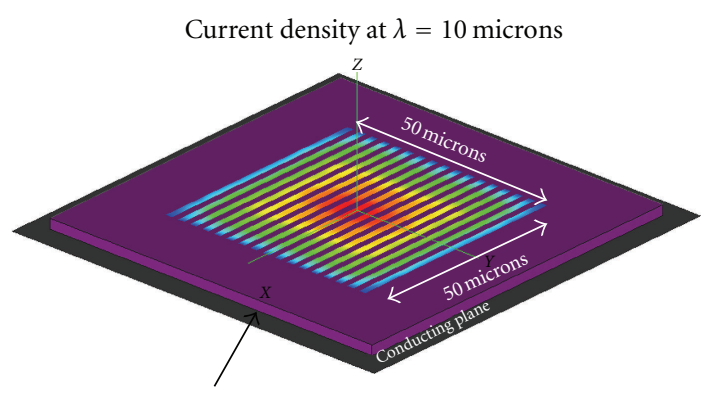

ZnSe thickness

2 microns

FIGURE 8: Current density distribution for a finite-sized resonant particle. Simulations were conducted using HFSS FEM solver.

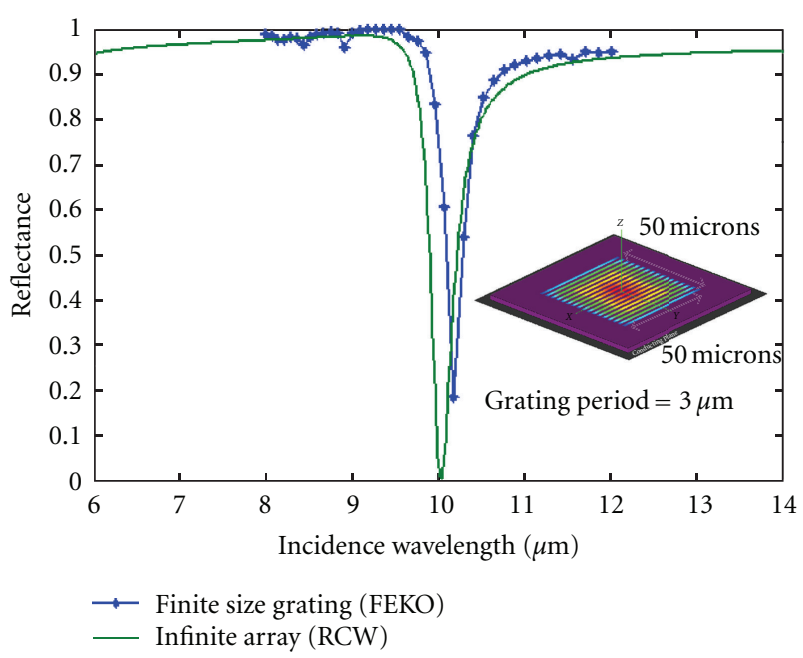

FIGURE 9: Predicted reflectance curves for a finite-sized particle compared to the infinitely periodic calculations. The edge effects of the finite-sized sample are evident but do not significantly alter the resonant peak.

base pressure of $1 \times 10^{-6}$. A 120 - $\AA$ layer of yttrium oxide $\left(\mathrm{Y}_{2} \mathrm{O}_{3}\right)$ was deposited first to promote adhesion between the substrate and the $\mathrm{ZnSe}$.

Photolithography on the ZnSe was achieved by first spin coating the wafer assembly with AZ \# 5214 image reversal photoresist at a speed of $4000 \mathrm{rpm}$ for 40 seconds. This photoresist was hot plate baked at $110^{\circ} \mathrm{C}$ for 2 minutes, exposed on a JBA vacuum contact aligner for 20 seconds with a bulb intensity of $4 \mathrm{~mW} / \mathrm{cm}^{2}$, hotplate baked (reversal bake) at $124^{\circ} \mathrm{C}$ for 40 seconds, and flood exposed for 25 seconds. The resist was then developed in AZ 300 MIF photoresist developer for 60 seconds and rinsed in deionized (DI) water for 1 minute. The wafer was then dried with nitrogen gas. The resulting photolithography was inspected under a microscope for clearing. Prior to loading the wafer assembly into the e-beam evaporator for the grating structure, a photoresist cleaning in a barrel plasma asher was performed. The patterned wafer assembly was placed into the vacuum ebeam evaporator and a metallization of $300 \AA$ titanium (Ti) followed by $1000 \AA$ of gold was completed. A metal liftoff 
using acetone, isopropyl, and DI water removed the excess metal. This fabrication process is graphically illustrated in Figure 10.

\section{Experimental Characterization}

Experimental characterization results for samples that were fabricated using the method described earlier are shown in Figures 11 and 12. For these samples, the ZnSe substrate thickness was fixed at $1.8 \mu \mathrm{m}$ and the linear gold gratings were spaced $3.0 \mu \mathrm{m}$ with a $50 \%$ duty cycle.

The IR reflectance and emission measurements were made using a Nicolet 560 Fourier transform infrared (FTIR) spectrometer with a near-normal incidence reflectivity module and an input port for collecting IR emission or photoluminescence. The reflectivity was taken at room temperature as a function of incident polarization. Although the polarized emission could be easily detected at room temperature, the signal-to-noise ratio was improved by taking the data at an elevated temperature. The experimental results, which closely match the modeled results, demonstrate a strong resonant absorption and thermal emission near the designed wavelength.

\section{Alternative Polarization Insensitive Designs}

One disadvantage of using the resonant particles described in Figure 2 is their sensitivity to polarization. This reduces the total absorbed energy by one half. To address this issue, we explored a number of designs that were less sensitive to incident field polarization. These structures, shown in Figure 13, consist of 2D arrays of gold strips (known commonly as a fishnet structure), metallic patches, and circular holes. Each of the structures shown in Figure 11 was analyzed using the RCW method. Of those structures analyzed, the inductive grid array (Figure 13(c)) showed the most promise. Figure 12 presents numerical simulations of normal incident reflectance as a function of wavelength. A strong, nearly perfect, absorption is predicted for both parallel and perpendicular polarization. Moreover, as in the previous designs, the resonant wavelength was easily tuned by simply varying the thickness of the dielectric substrate layer. It should be noted that the results shown in Figure 14 have not been experimentally validated yet.

\section{Conclusions}

In this paper, we presented a design methodology to create small particles characterized by a strong resonant absorption within the LWIR $(8-14 \mu \mathrm{m})$ band. Our method combined a surface plasmon resonance, created using a subwavelength metallic grating with a dielectric cavity resonance. We showed that by varying the thickness of the cavity substrate the resonances could be tuned anywhere within the LWIR band. Experimental samples were fabricated using photolithography and experimentally characterized. The experimental results compared favorably with the calculated results. We believe that material-by-design particles, such

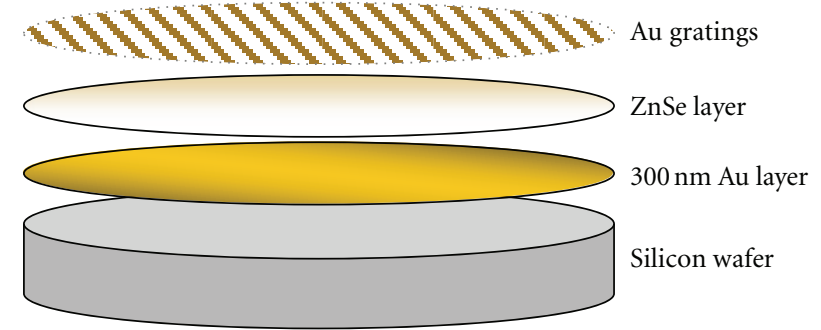

FIgURE 10: Illustration of the fabrication steps.

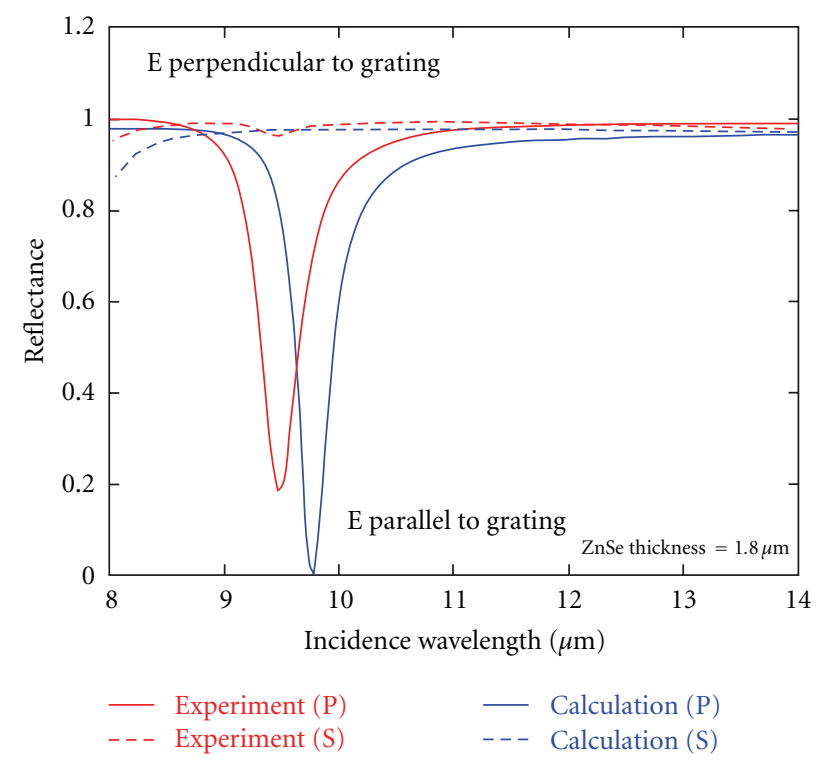

FIgure 11: A comparison of predicted (using RCW code) and measured reflectance for both parallel (P type) and perpendicular (S type) polarizations.

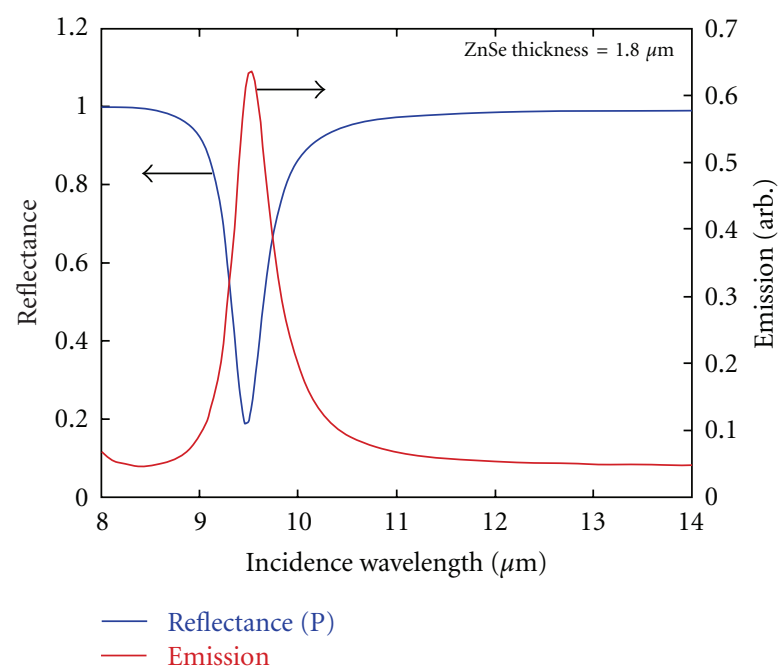

FIGURE 12: Experimentally measured reflectance curve and emission curve clearly demonstrating the resonant nature of our design. 


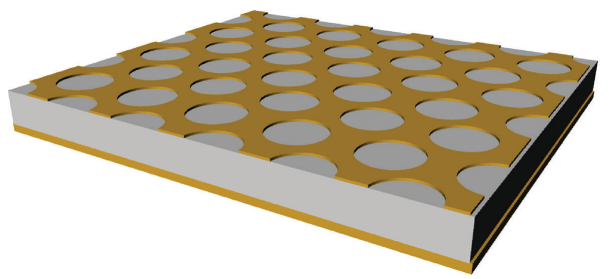

(a) Hole array

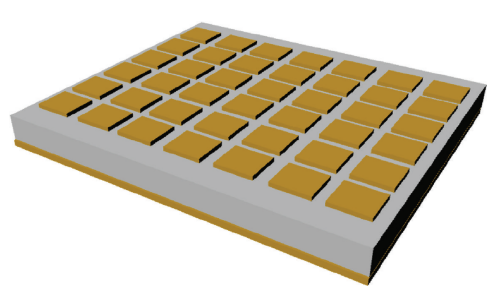

(b) Capacitive patch array

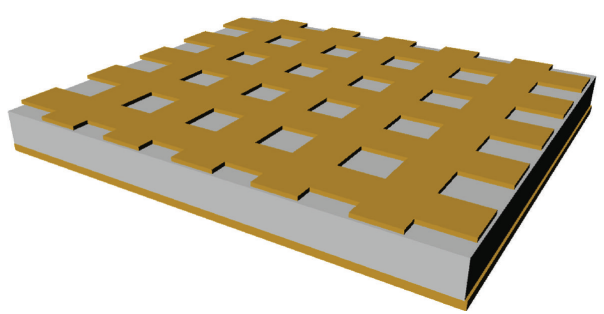

(c) Inductive grid array (fishnet)

Figure 13: Designs less sensitive to polarization effects.

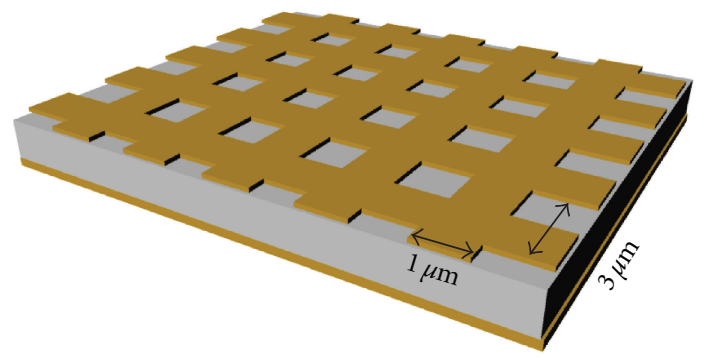

(a)

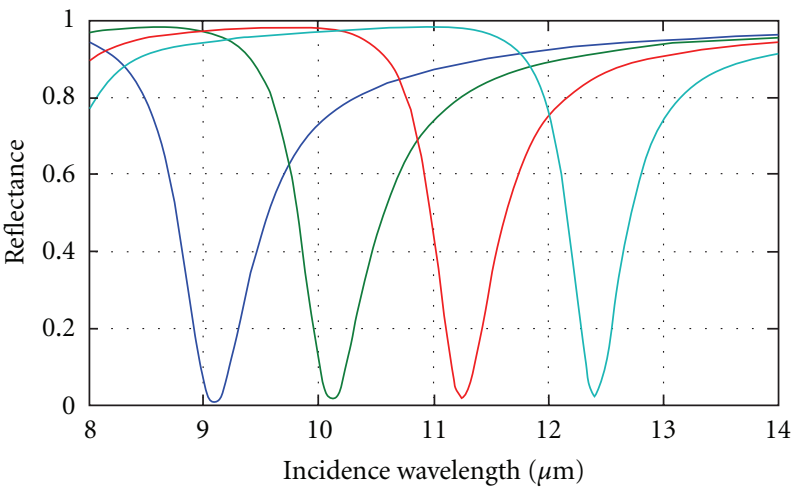

Substrate thickness $\begin{array}{ll}- & 1.75 \text { microns } \\ - & 2 \text { microns }\end{array}$
- 2.25 microns

- 2.5 microns

(b)

Figure 14: RCW simulations for inductive grid array shown in Figure 11(c). Here as the substrate, assumed to be ZnSe, is varied from 1.75 to $2.5 \mu \mathrm{m}$. The resonant absorption wavelength shifts to longer wavelengths; however, the magnitude of the absorption remains near perfect.

as the ones described here, would be useful for a variety of remote atmospheric sensing applications. In those applications, which require relatively small particles, a custom spectral signature with multiple wavelengths would be achieved by mixing batches of single-wavelength particles designed for the component wavelengths. But in other applications, such as calibrated surfaces for hyperspectral imager testing and training, the surfaces could be larger and the multiple wavelengths could be designed into a single surface by implementing a checkerboard subcells with different grating periods across the surface. By properly selecting the frequencies and relative areas of the emitting subcells, the emission spectrum could be designed to mimic the spectral emission from specific natural surfaces.

\section{References}

[1] K. Masuno, S. Kumagai, and M. Sasaki, "Reflection-type wavelength-selective infrared emitter using surface plasmon polaritons," Optics Letters, vol. 36, no. 3, pp. 376-378, 2011.

[2] J. Hendrickson, J. Guo, B. Zhang, W. Buchwald, and R. Soref, "Wideband perfect light absorber at midwave infrared using muliplexed metal structures," Optics Letters, vol. 37, no. 3, pp. 371-373, 2012.

[3] K. Boratay Alici, A. Burak Turhan, C. M. Soukoulis, and E. Ozbay, "Optically thin composite resonant absorber at the near-infrared band: a polarization independent and spectrally broadband configuration," Optics Express, vol. 19, no. 15, pp. 14260-14267, 2011.

[4] N. Liu, M. Mesch, T. Weiss, M. Hentschel, and H. Giessen, "Infrared perfect absorber and its application as plasmonic sensor," Nano Letters, vol. 10, no. 7, pp. 2342-2348, 2010.

[5] C. H. Lin, R. L. Chern, and H. Y. Lin, "Polarizationindependent broad-band nearly perfect absorbers in the visible regime," Optics Express, vol. 19, no. 2, pp. 415-424, 2011.

[6] P. Lalanne and J. P. Hugonin, "High-order effective-medium theory of subwavelength gratings in classical mounting: application to volume holograms," Journal of the Optical Society of America A, vol. 15, no. 7, pp. 1843-1851, 1998.

[7] M. G. Moharam and T. K. Gaylord, "Rigorous coupled-wave analysis of planar-grating diffraction," Journal of the Optical Society of America, vol. 71, no. 7, pp. 811-818, 1981.

[8] P. Lalanne, "Improved formulation of the coupled-wave method for two-dimensional gratings," Journal of the Optical Society of America A, vol. 14, no. 7, pp. 1592-1598, 1997.

[9] E. Noponen and J. Turunen, "Eigenmode method for electromagnetic synthesis of diffractive elements with threedimensional profiles," Journal of the Optical Society of America A, vol. 11, no. 9, pp. 2494-2502, 1994.

[10] P. G. Etchegoin, E. C. Le Ru, and M. Meyer, "An analytic model for the optical properties of gold," Journal of Chemical Physics, vol. 125, no. 16, Article ID 164705, 3 pages, 2006. 

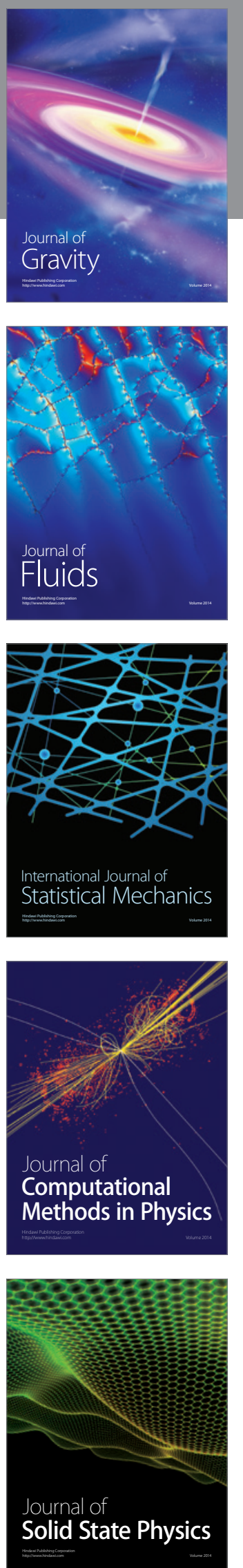

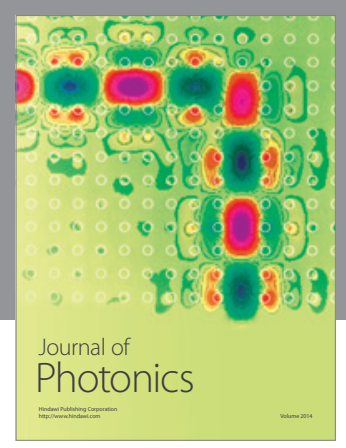

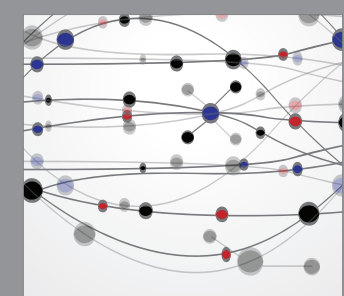

The Scientific World Journal
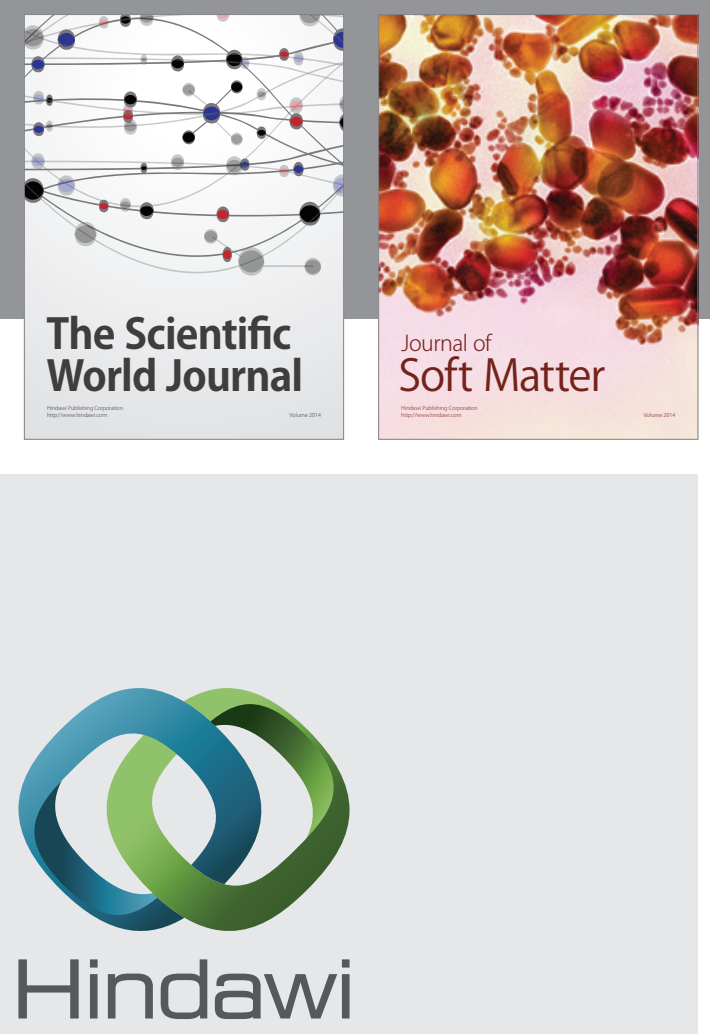

Submit your manuscripts at

http://www.hindawi.com
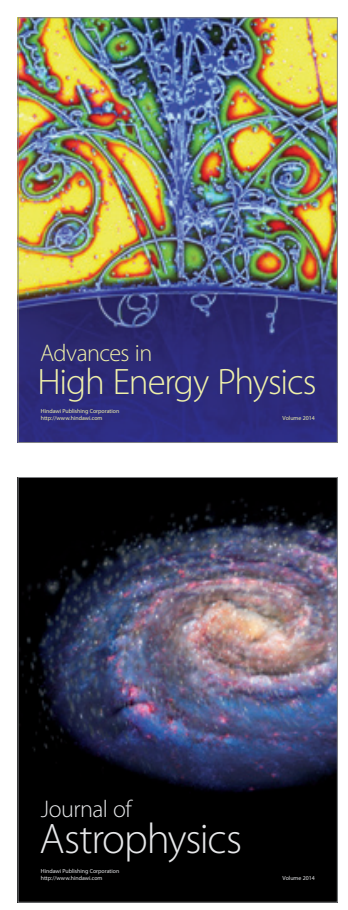
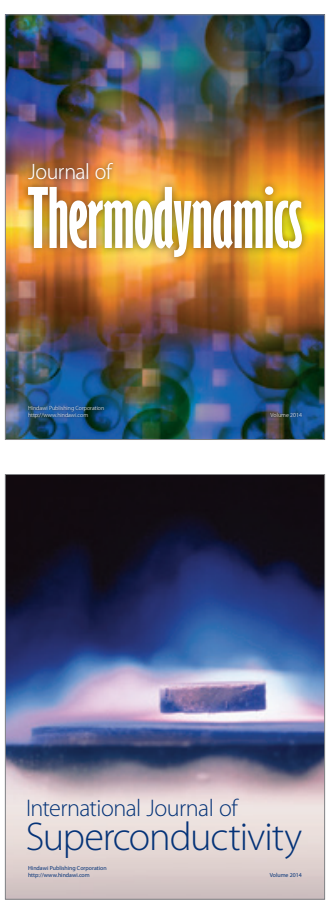
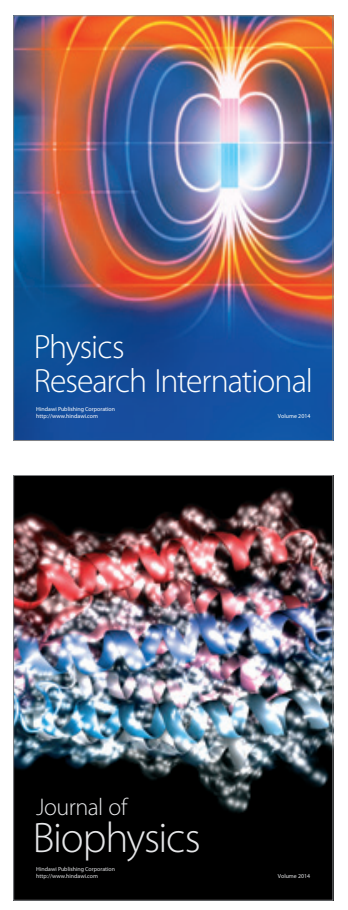
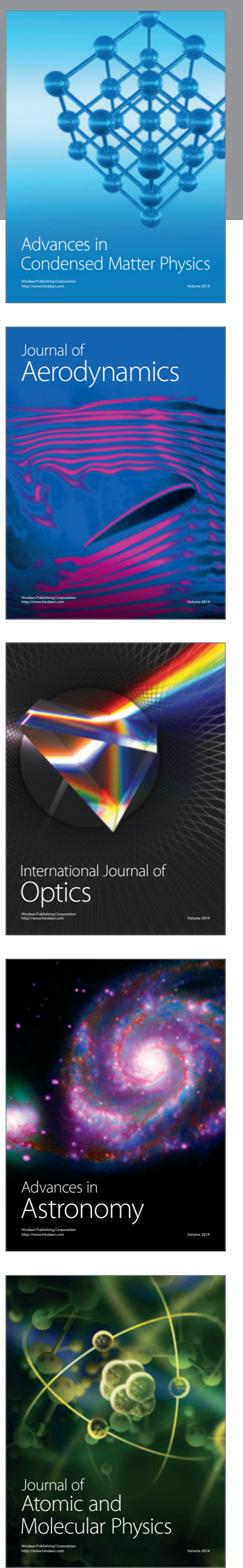